\title{
Lighting up the path to romance
}

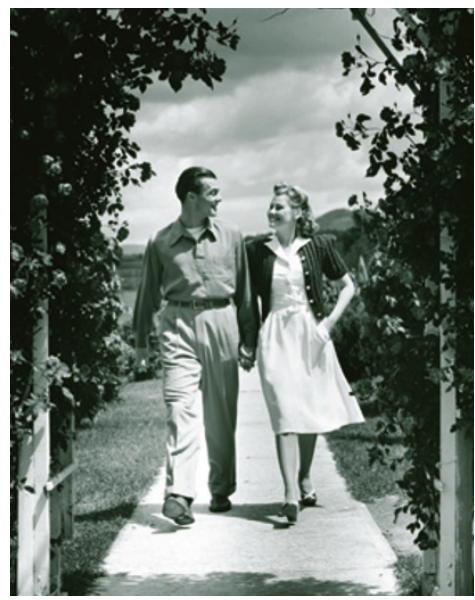

In Drosophila males, the pheromone cis-vaccenyl acetate (cVA) suppresses mating with other males, whereas in females it promotes receptivity to males. Axel and colleagues now show that the neural circuit that cVA activates differs subtly between males and females, which could explain how this single pheromone can promote such different behaviours.

In both sexes, receptors for cVA are expressed in a subset of olfactory sensory neurons that project to the DA1 glomerulus in the antennal lobe. Here these neurons synapse with projection neurons that innervate the lateral horn of the protocerebrum, which regulates the initiation of courtship behaviour. Both cVAreceptor-containing olfactory neurons and their associated DA1 projection neurons express fruitless (fru), an important regulator of courtship behaviour.

The authors first characterized the electrophysiological properties of fru-expressing DA1 projection neurons and showed that these neurons respond similarly to cVA in male and female flies, indicating that the sexually dimorphic behavioural effect of cVA cannot be explained by different electrophysiological responses to the pheromone.

To investigate whether the sexspecific effects of cVA might instead be due to sex differences in the neural circuit that it activates, Axel and colleagues expressed a photoactivatable green fluorescent protein (PA-GFP) specifically in fru-expressing neurons. Photoactivation of PA-GFP through targeted illumination of DA1 projection neurons then allowed the authors to visualize individual projection neurons' innervation paths to the lateral horn.

The authors observed that the ventral branches of the axonal projections of fru-expressing DA1 neurons in the lateral horn were denser in males than in females. They then investigated whether sex-specific splice variants of fru regulate this sexually dimorphic projection pattern. Indeed, the density of DA1 axons' ventral branches was lower in fru-mutant males than in wild-type males. Conversely, in females expressing the malespecific form of $f r u\left(f r u^{\mathrm{M}}\right)$, the DA1 axon arbour resembled that of males, although this was not the case if $f r u^{\mathrm{M}}$ was expressed specifically in DA1 projection neurons. Taken together, these data indicate that $f r u^{\mathrm{M}}$ expression in DA1 projection neurons and in other yet to be identified fruexpressing neurons is required for male-specific ventral axon arborization in the lateral horn.

The sexually dimorphic neural circuit identified by the authors provides a likely mechanism by which cVA induces divergent mating behaviours. Moreover, this study has shown that photoactivatable protein expression provides a sophisticated method for tracing individual neurons in the living brain.

\section{Leonie Welberg}

ORIGINAL RESEARCH PAPER Datta, S. R. et al. The Drosophila pheromone cVA activates a sexually dimorphic neural circuit. Nature $27 \mathrm{Feb}$ 2008 (doi:10.1038/nature06808) 